\title{
LES of Mixed Convection Boundary Layer Between Radiating Parallel Plates
}

\author{
D. G. Barhaghi ${ }^{1}$, L. Davidson ${ }^{1}$ \\ ${ }^{1}$ Division of Fluid Dynamics, Department of Applied Mechanics, \\ Chalmers University of Technology, SE-412 96 Göteborg, Sweden, \\ darioush@chalmers.se and lada@chalmers.se
}

\begin{abstract}
The influence of natural convection on a mixed convection boundary layer in a vertical channel is studied using Large-eddy simulation. A constant heat flux boundary condition is applied to one of the channel walls while the other wall is insulated. The Grashof and Reynolds numbers based on the wall heat flux and the channel width are $G r=9.4 \cdot 10^{7}$ and $R e=5080$. As the temperature difference between the hot wall and the inlet air is large, the governing equations are solved using two different approaches namely Boussinesq approximation considering constant properties and the equations with variable properties. As a consequence of hot wall high temperature, radiation is also taken into account and it is shown that about $10 \%$ of the heat is transferred via radiation. Although variable property equations yield better solutions compared to the constant property equations, there exist substantial differences between the computed and the measured profiles which might be due to the numerical scheme that has impaired the imposed turbulence at the inlet.
\end{abstract}

\section{Introduction}

Natural convection heat transfer exists in a number of industrial applications including reactors, and heat exchangers. An extensive study of this kind of boundary layer including both experiments and numerical simulations can be found in the literature. The results of these investigations have shown substantial differences between the conventional forced convection and natural convection boundary layers. However, there are still some aspects on which there is no general consensus among the researchers. On the other hand, the influence of natural convection in case of mixed convection boundary layers for moderate ratios of Reynolds to Grashof number is believed to be crucial. So appropriate modeling of this type of boundary layer demands further understanding of this flow (see [1]).

Pure natural convection boundary layer on a vertical flat plate is studied experimentally in [2]. A unique feature of this flow is the production of turbulence in the region close to the wall which has large influence on the turbulent flow regime in the vicinity of the wall. In forced convection boundary layers, it is the Reynolds shear stress which contributes to the production of turbulence, whereas in natural convection boundary layer, it can be a sink term in the inner part of the boundary layer where the velocity gradient is positive. Consequently, the buoyancy production takes over the role of the source term and compensates for the production deficit due to the Reynolds shear stress. However, the results of the LES of natural convection boundary layer on a vertical cylinder in [3] does not completely comply 
with the findings of the mentioned experiment in the region close to the wall.

In order to appropriately model the turbulent flows to which the contribution of the natural convection is important, the length scale over which buoyancy expresses itself should be well understood. By assuming different level of interactions between the buoyancy and turbulent momentum, different modeling approaches can be considered. Study of the effect of the buoyancy on the turbulence budget of mixed convection boundary layer in a vertical infinite channel in [4] shows that the buoyancy is only an important term in the momentum equation but not the stress equations. Mixed convection boundary layers in the presence of radiative heat transfer is also investigated in [5]. This work brought the motivation for further analysis of the turbulence in a mixed convection boundary layer and the effect of the buoyancy especially in comparison with the case of infinite channel. In the latter flow the boundary layer is fully developed and no stream-wise advective heat transfer exists.

\section{Geometry and Grid Configuration}

Figure 1 represents the buoyancy aided channel flow configuration which is in accordance to the experimental configuration in [5]. Two different grids with resolu-

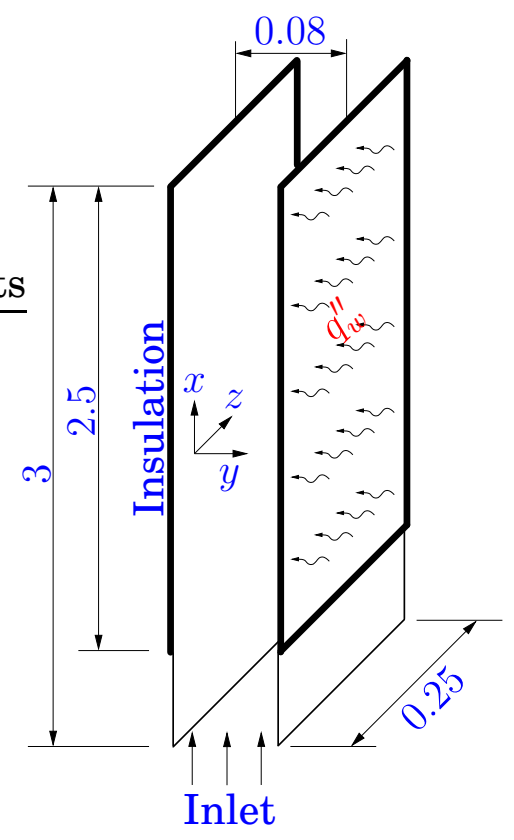

Figure 1: Computational geometry configuration. All the dimensions are in meter.

tions of $322 \times 66 \times 50$ (hereafter coarse grid) and $482 \times 66 \times 66$ (hereafter fine grid) in the $x, y$ and $z$ directions, respectively, are used to carry out the computations. The spatial resolution for the coarse grid, along the hot wall, is $\Delta z^{+}<45$ and $\Delta x^{+}<80$ in the span-wise and stream-wise directions. For the fine grid, the corresponding values are $\Delta z^{+}<33$ and $\Delta x^{+}<50$. In both cases $y^{+}<0.6$ for the wall adjacent node and $9 \%$ stretch factor is used in the wall-normal $(y)$ direction. 


\section{Numerical Method}

Applying constant heat flux boundary condition on the hot wall causes the temperature of the hot wall to raise in the stream-wise direction. From the primary computations, it was found that the temperature difference between the hot wall and the inlet air raises to as high as $100^{\circ} \mathrm{C}$. The first consequence of the high temperature difference in the flow is the ambiguity of the Boussinesq approximation validity. Thus, the Navier-Stokes and the energy equations are not only solved using Boussinesq approximation, but they are solved considering variable properties including varying density, viscosity, conductivity and specific heat. All the properties are extracted from thermodynamical tables and fourth order polynomial equations are fitted on them assuming they are independent of pressure. These equations are used in the finite volume code in order to estimate the properties.

\subsection{Finite volume approach}

A conventional finite volume method is used to solve the three-dimensional continuity, Navier-Stokes and temperature equations with the above mentioned characteristics. Space filtering is applied to the equations with constant properties. However, Favre averaged equations are solved in the case with the variable properties. The dynamic subgrid-scale model of [6] is used to model the small eddies. In order to avoid the spurious fluctuations that occur in cells with the Peclet number $|P e|>2$, a blend of the central difference scheme with deferred correction (see [7]) and Van-Leer scheme in conjunction with a wiggle detector is used to discretize the equations in space. Second-order Crank-Nicolson scheme is used in time.

The numerical procedure is based on an implicit, fractional step technique with a multi-grid pressure Poisson solver and a non-staggered grid arrangement. The pressure Poisson equation in case of the constant property equations reads:

$$
\frac{\partial^{2} P}{\partial x_{i} \partial x_{i}}=\frac{\rho}{\Delta t} \frac{\partial u_{i}^{*}}{\partial x_{i}}
$$

where $u_{i}^{*}$ is the intermediate velocity in the fractional step solution procedure (see [8] for details). In the variable property case, considering the continuity equation, the Poisson equation for pressure reads:

$$
\frac{\partial^{2} P}{\partial x_{i} \partial x_{i}}=\frac{1}{\Delta t}\left(\frac{\partial \rho}{\partial t}+\frac{\partial \rho u_{i}^{*}}{\partial x_{i}}\right)
$$

\subsection{Boundary conditions}

As it is depicted in Figure 1 the channel left wall is kept insulated for $x>0.5 \mathrm{~m}$. At the right wall, constant heat flux boundary condition, $q_{w}^{\prime \prime}=449 \mathrm{~W} / \mathrm{m}^{2}$, is applied. Air temperature and the Reynolds number are $T_{i n}=18.6^{\circ} \mathrm{C}$ and $R e_{W}=5080$. The Grashof number is $G r_{W}=g \beta q_{w}^{\prime \prime} W^{4} /\left(\nu^{2} k\right) \approx 9.4 \cdot 10^{7}$ where $W=0.08 \mathrm{~m}$. So, the Grashof to Reynolds number ratio is $G r_{W} / R e_{W}^{2} \approx 3.6$, suggesting that the buoyancy effects are as important as the inertial effects.

The instantaneous DNS results of a fully developed channel are prescribed as the inlet boundary condition. At the outlet, convective boundary condition has been 
used for velocities and temperature. Cyclic boundary condition is applied in the span-wise $(z)$ direction.

Another consequence of the hot wall high temperature is that the radiation heat transfer becomes significant. The radiation heat transfer is assumed to be twodimensional meaning that span-wise variation of the radiation is neglected. Provided the radiosity equation for each grid cell on the channel walls, the GaussSeidel method is used to solve for the surface radiosities at each time step. The emissivity of the channel walls is set to $\varepsilon=0.125$ (from the experimental data) and both inlet and outlet are assumed to be black body surfaces.

\section{Results}

After assessing fully developed flow condition by tracking the time variation of the Nusselt number at different heights, sampling is started. In the constant property case, time averaged samples are obtained which is defined as $\langle\phi\rangle=1 / T \int_{0}^{T} \phi$. In the variable property case, however, Favre averaged samples are obtained which by considering time averaging definition is $\widetilde{\phi}=\langle\rho \phi\rangle /\langle\rho\rangle$. In the following, unless otherwise stated, the results of the case with the fine grid considering variable properties and including radiation are presented.

\subsection{Wiggle detector and inlet boundary condition}

To the authors surprise, wiggle detector is found to have severe impact on the inlet boundary condition. It turned out that although this method prohibits the spurious fluctuations to grow, it has a major disadvantage of damping the imposed turbulence at the inlet. This can be seen in Figure 2(a) which shows the averaged proportion of the Van-Leer and central difference schemes. In the figure, one and zero correspond to pure central difference and pure Van-Leer schemes, respectively. Except for the inlet, wiggle detector has mostly used the central difference scheme.

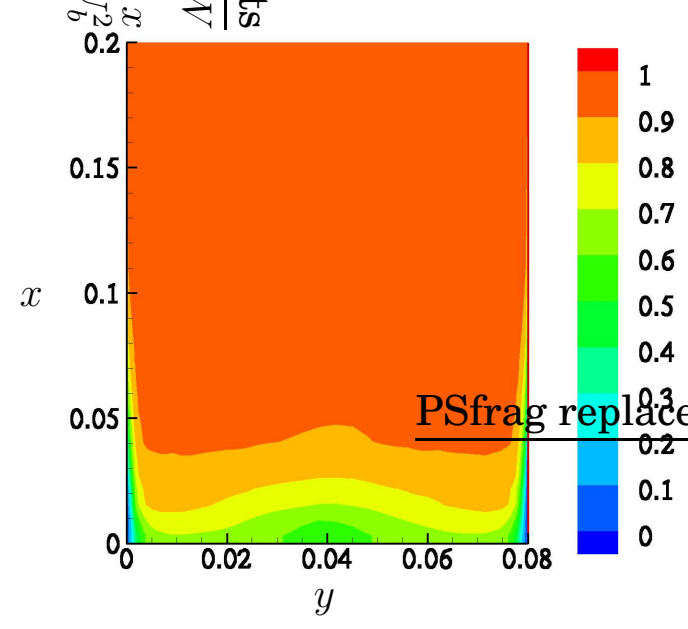

(a) Contours of scheme usage ratio

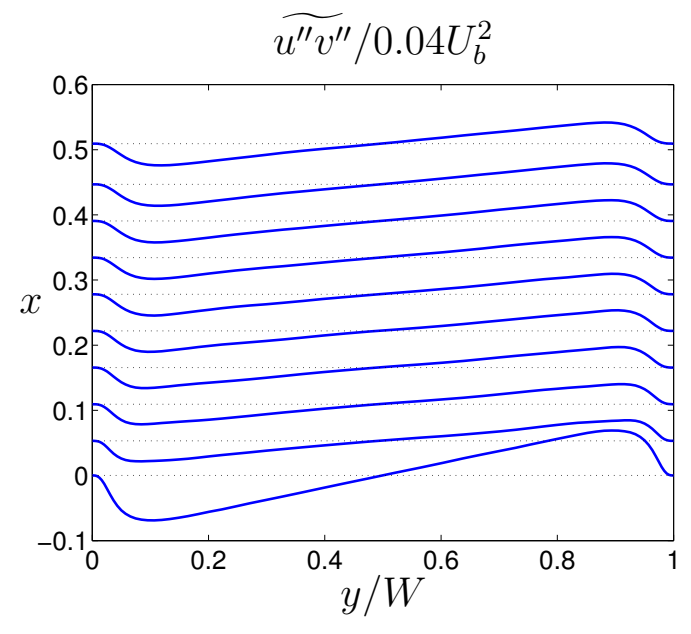

(b) Turbulent shear stress variations

Figure 2: The average proportion of the schemes used by wiggle detector and the shear stress near the inlet for the fine mesh and variable properties. 
This can also be understood from Figure 2(b) that depicts the results of the shear stress. It is clear that the shear stress is preserved at $x>0.05 \mathrm{~m}$. It can be hypothesized that the turbulence at the inlet is partly incoherent with respect to the flow in the channel.

\subsection{Grid dependency assessment}

Figure 3 compares the velocity and the temperature profiles for different grid resolutions, considering constant fluid properties. Although velocity profiles show al-

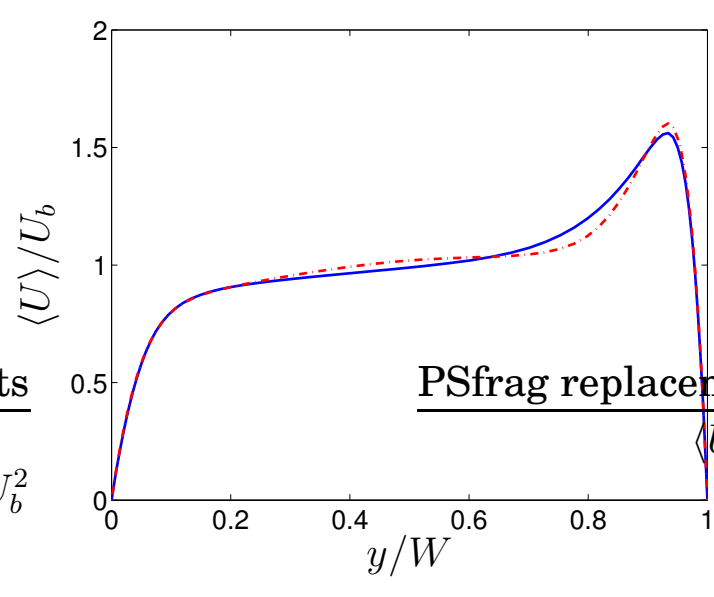

(a) Normalized velocity profile

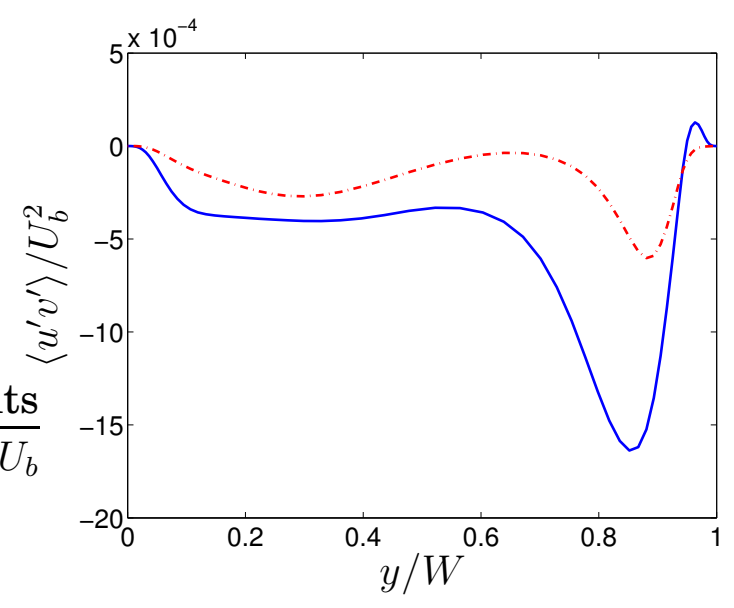

(b) Turbulent shear stress

Figure 3: Velocity and turbulent shear stress profiles at $x=2.5 \mathrm{~m}$ for constant property computations. _ :fine mesh; ... : coarse mesh.

most the same behavior for different grids, turbulent shear stress profiles are significantly different.

Results of the variable property computations is also shown in Figure 4. Unlike the constant property case, the results of the variable property computations show less grid dependency for both mean velocity and turbulent shear stress owing to density fluctuations that prevents oscillations by affecting the wiggle detector.

\subsection{Property dependency assessment}

Variable properties assumption leads to slower convergence rate. In order to find whether or not the Boussinesq approximation is accurate enough, the mean velocity and the turbulent shear stress are compared against the experimental results in Figure 5. Figure 5(a) shows a thicker boundary layer in case of the variable property assumption which is connected to the thicker boundary layer and yields larger shear stress (see Figure 5(b)). Close to the insulated wall, both the variable and constant property assumptions yield the same velocity and shear stress distribution proving that Boussinesq approximation is accurate enough for small temperature differences. However, even the variable property results deviate from the measurements in the region close to the hot wall. The measured velocity distribution, resembles a fully developed channel flow velocity distribution in the existence 


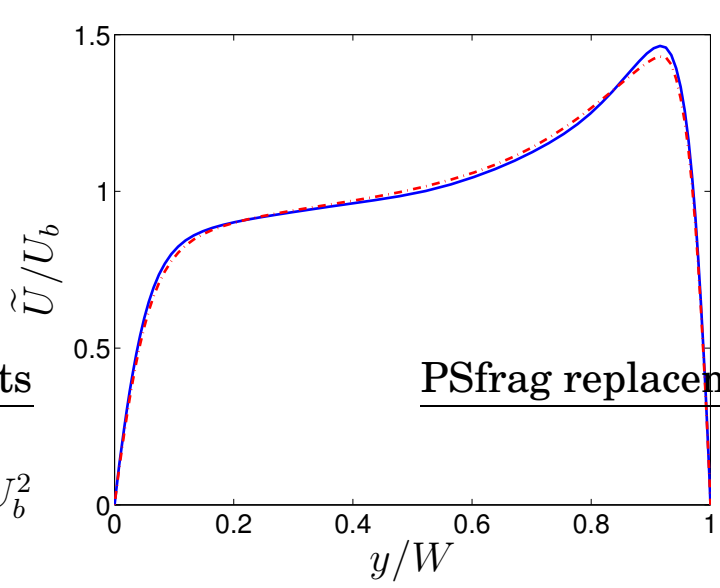

(a) Normalized velocity profile

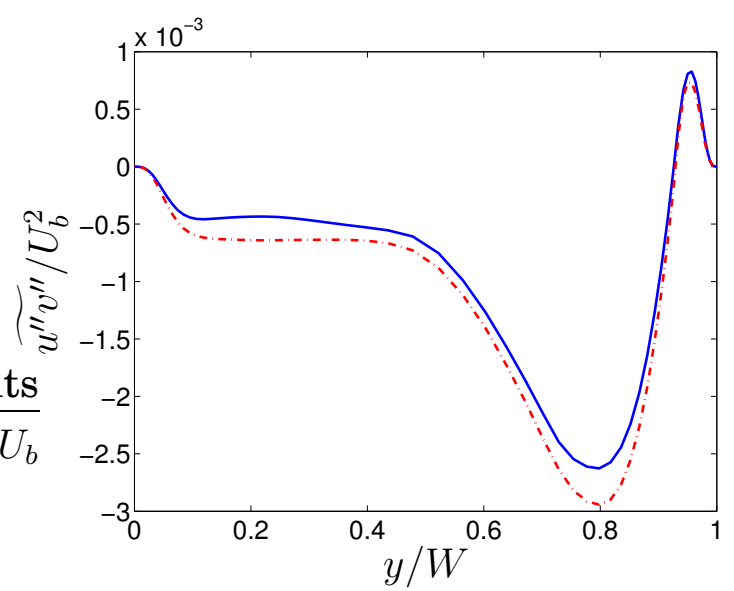

(b) Turbulent shear stress

Figure 4: Velocity and turbulent shear stress profiles at $x=2.5 \mathrm{~m}$ for variable property computations. — :fine mesh; -.. : coarse mesh.

of buoyancy (see [4]) which is not the case for the computations. Presumably, this is due to dampened fluctuations by the wiggle detector at the inlet.

\subsection{Radiation importance}

Radiation is calculated at each time step and it is added as a sink/source term in the cells adjacent to the hot/insulated wall. Figure 6(a) compares the velocity profiles of two cases where the radiation heat transfer is considered and neglected, respectively. In both cases, it is assumed that the flow properties remain constant and computations are run on the coarse grid.

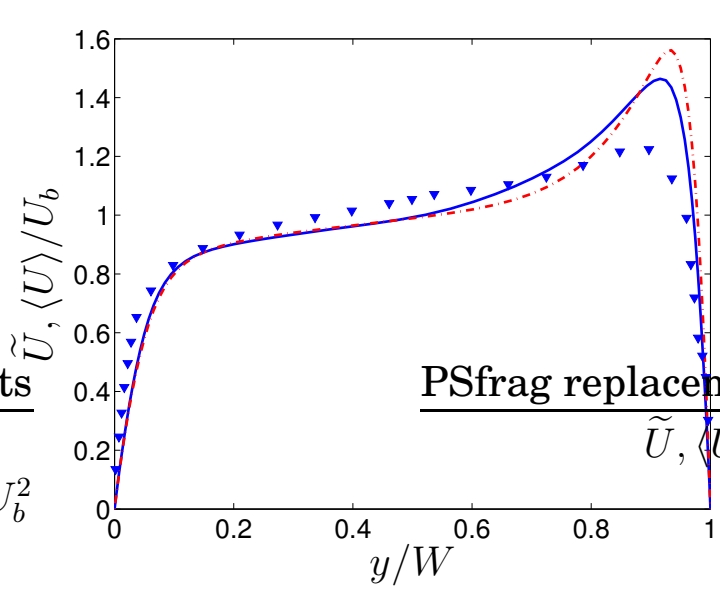

(a) Normalized velocity profile

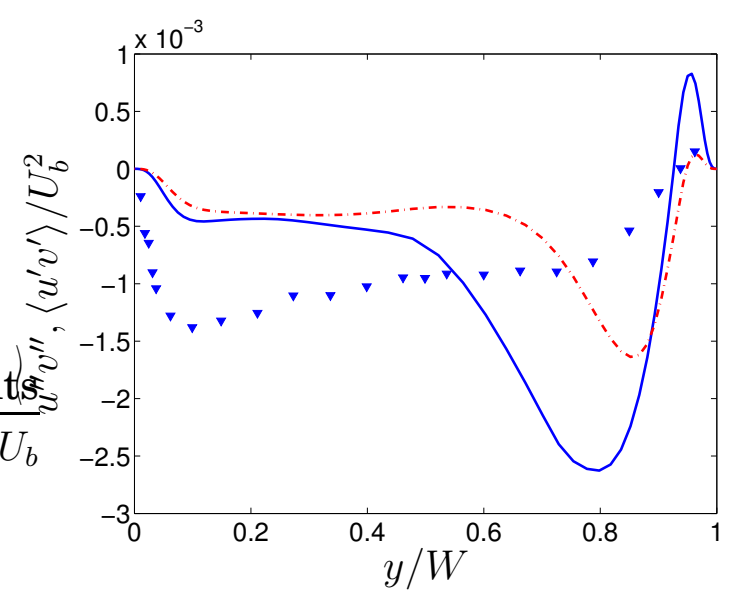

(b) Turbulent shear stress

Figure 5: Variable and constant property results comparison at $x=2.5 \mathrm{~m}$. — :variable property; ... : constant property; $\nabla$ :Experimental results of [5]. 


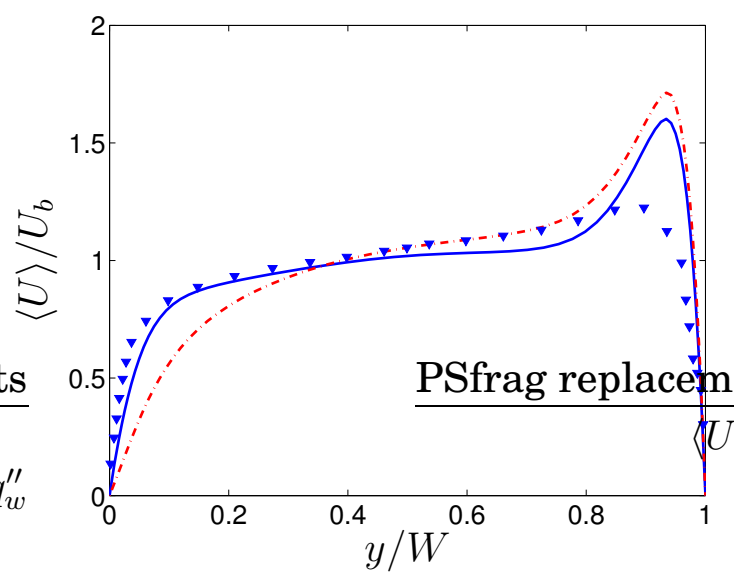

(a) Velocity profile.__ : with radiation; - - - :neglecting radiation; $\nabla$ :measurements

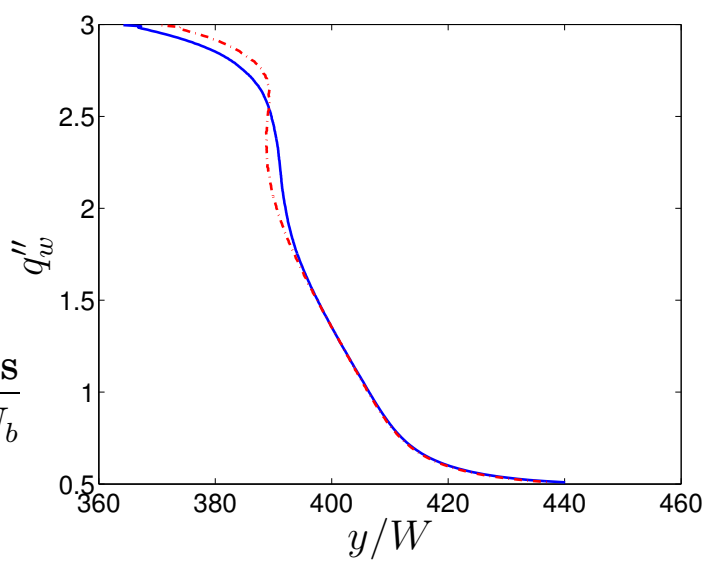

(b) Hot wall heat flux._ :Fine grid; - - :coarse grid

Figure 6: Effect of radiation heat transfer on velocity and the remaining heat flux on the hot wall.

Figure 6(b) shows the conductive hot wall heat flux on both fine and coarse grids considering variable properties. Since the total heat flux on the hot wall is $449 \mathrm{~W} / \mathrm{m}^{2}$, on average more than $10 \%$ of the heat flux is transferred via radiation. Therefore, the radiation heat transfer is an important aspect of this problem and can not be neglected.

\subsection{Mean flow parameters}

Velocity profiles were shown in the previous sections. Another two important parameters are temperature and Nusselt number whose variations are shown in Figure 7. Once again, the effect of the radiation can be seen in Figure 7(a) in which the variation of the temperature along the insulated wall is shown. This temperature would remain constant, equal to the inlet temperature $\left(T_{b}=18.6^{\circ} \mathrm{C}\right)$, if the effect of radiation was neglected. The reason why temperature falls slightly at $x>2.5 \mathrm{~m}$ is that the outlet is considered to be a black body surface. Hence, it is assumed that each cell on the walls exchanges heat with outlet via radiation assuming the outlet temperature to be equal to the bulk temperature at the outlet.

Figure 7(b) compares the variation of the computed and measured Nusselt numbers along the hot wall for $x>0.5 \mathrm{~m}$. The predicted Nusselt number is smaller than the measured one. This difference is especially large in the lower part of the channel where $0.5<x<0.8$. This large difference is again due to low predicted turbulence intensity at the entrance region which is caused by the wiggle detector and the Van-Leer scheme.

\subsection{Reynolds stresses}

Aside from the Reynolds shear stress which is shown above, all other Reynolds normal stresses, turbulent heat fluxes and temperature fluctuations are plotted 


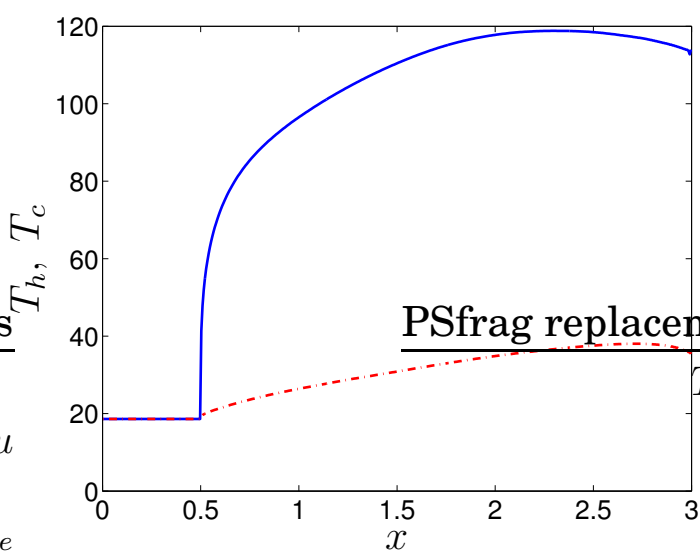

(a) Temperature variations.__ : Hot wall; _ _ _ :insulated wall.

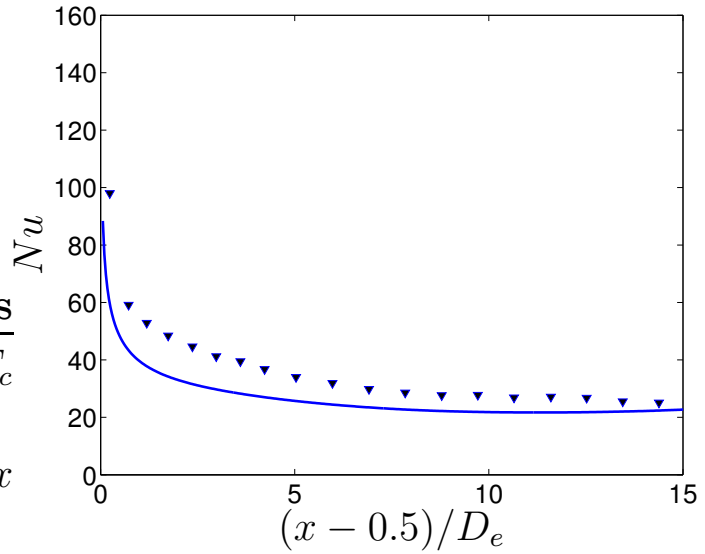

(b) Nusselt number.__ :Computations; $\nabla$ :measurements. $D_{e}=2 \cdot W$

Figure 7: Temperature variations along the hot and insulated wall and Nusselt number variations along the hot wall considering variable properties and radiation.

in Figure 8. Similar to the turbulent shear stress, all other computed Reynolds stresses as well as turbulent heat fluxes and temperature fluctuations show the behavior of a developing mixed convection boundary layer. However, the measured parameters show the behavior of a fully developed boundary layer. That is why the computed data show a reversed trend with respect to the measured data.

In case of the temperature fluctuations and turbulent heat transfer, no experimental data are available for comparison.

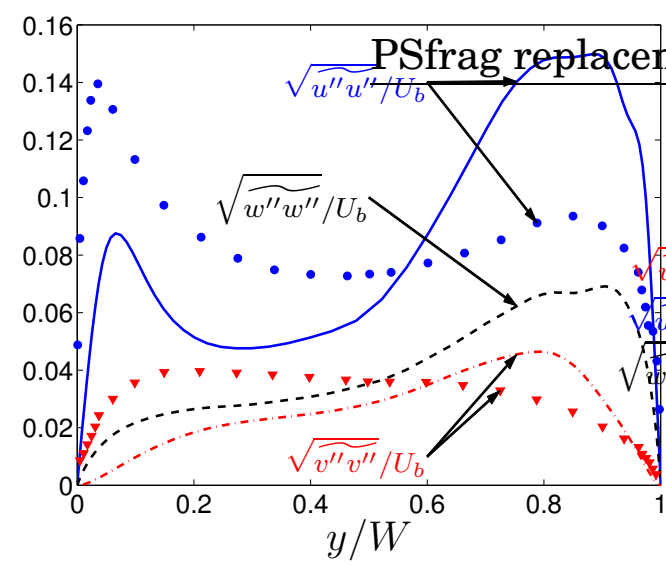

(a) Normal fluctuations.

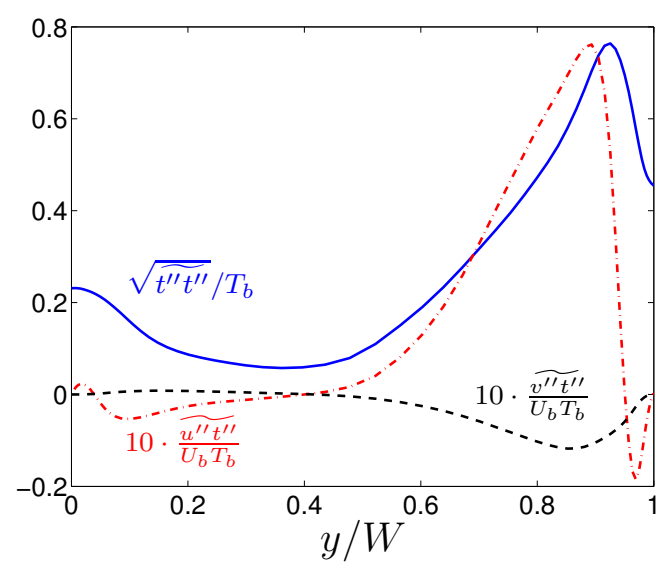

(b) Turbulent heat fluxes and temperature fluctuations.

Figure 8: Reynolds stresses, turbulent heat fluxes and temperature fluctuations at $x=2.5 \mathrm{~m}$. 


\section{Boundary Layer Development}

The computed turbulence and mean flow parameters shown in the previous sections suggest that the predicted boundary layer is still developing (due to the wiggle detector which damps the resolved fluctuations near the inlet) contrary to the experimental measurements. This can also be investigated by considering the velocity and temperature profiles as they develop along the channel passage. Velocity and temperature profiles at different heights are shown in Figure 9.

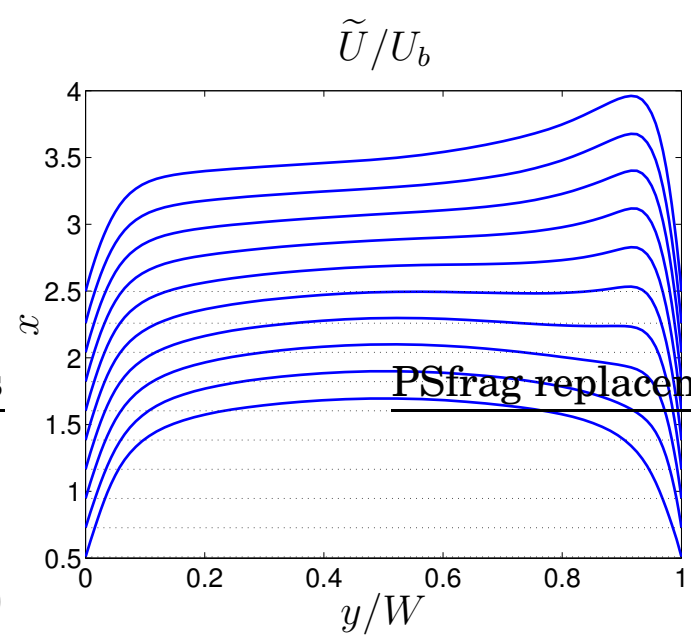

(a) Velocity profiles.

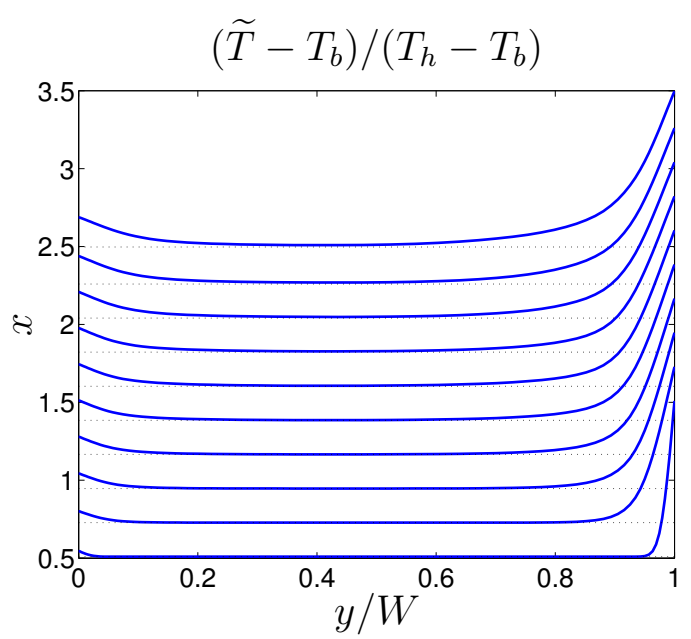

(b) Temperature profiles.

Figure 9: Velocity and temperature profiles across the channel at different heights.

The two important parameters that play an essential role in the turbulent boundary layer development are the turbulent shear stress $\left(\widetilde{u^{\prime \prime} v^{\prime \prime}}\right)$ and the wall normal turbulent heat flux $\left(\widetilde{v^{\prime \prime} t^{\prime \prime}}\right)$ which are shown in Figure 10. At $x=0.5 \mathrm{~m}$ where the heat flux boundary condition is applied, symmetrical profile of a fully developed channel flow can be observed in case of the turbulent shear stress. Since temperature boundary layer is just about forming, $\widetilde{v^{\prime \prime}} t^{\prime \prime}$ is zero at this height. Near the hot wall, as the boundary layer grows, $\widehat{u^{\prime \prime} v^{\prime \prime}}$ is reduced. As the flow goes upward, $\widetilde{u^{\prime \prime} v^{\prime \prime}}$ in the inner part of the boundary layer $(y / W>0.9)$, eventually becomes zero from where it starts to attain positive values. However, in the outer part of the boundary layer, the negative shear stress attains larger magnitudes since the production of shear stress, $P_{u v}=-\widetilde{v^{\prime \prime} v^{\prime \prime}} \partial \widetilde{U} / \partial y+g \beta \widetilde{v^{\prime \prime} t^{\prime \prime}}$, is negative (due to positive velocity gradient and negative wall normal heat flux). But, in the inner part of the boundary layer, the production of shear stress due to $\widehat{v^{\prime \prime} v^{\prime \prime}}$ is larger than the other negative term resulting in positive shear stress in the inner boundary layer.

By considering the major production term of the wall normal turbulent heat flux, $P_{v t}=-\widetilde{v^{\prime \prime} v^{\prime \prime}} \partial \widetilde{T} / \partial y$, it can be concluded that due to the positive temperature gradient and consequently negative $P_{v t}, \widetilde{v^{\prime \prime} t^{\prime \prime}}$ attains negative values close to the hot wall.

Since $\widetilde{v^{\prime \prime} v^{\prime \prime}}$ plays an important role in the productions of $\widetilde{u^{\prime \prime} v^{\prime \prime}}$ and $\widetilde{v^{\prime \prime} t^{\prime \prime}}$, its evolvement as the boundary layer grows is shown in Figure 11(a), $\widetilde{v^{\prime \prime} v^{\prime \prime}}$ is symmetrical at 


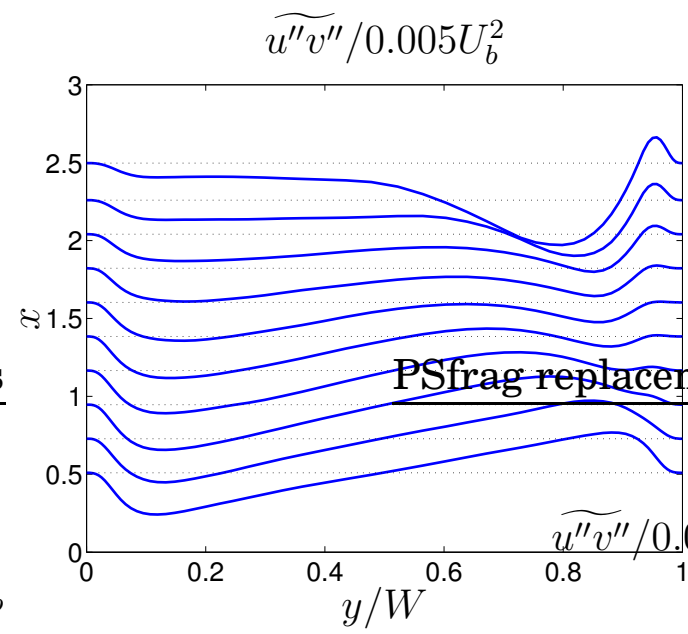

(a) Turbulent shear stress.

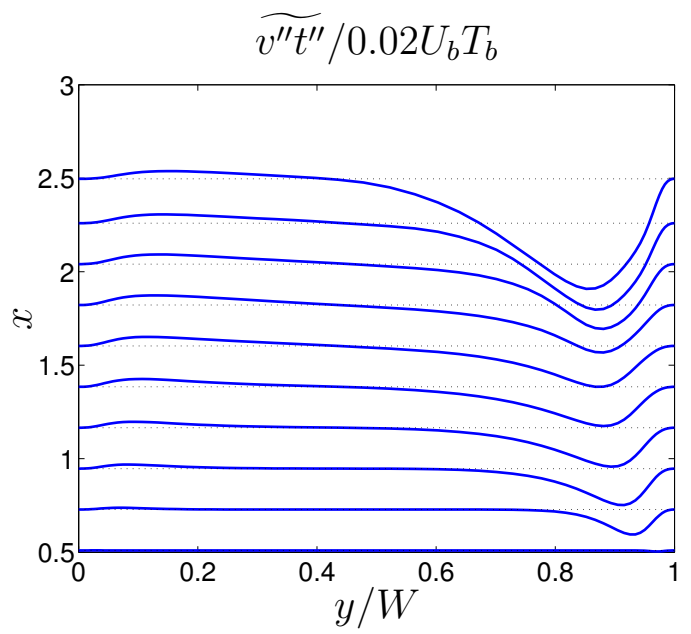

(b) Wall normal turbulent heat flux.

Figure 10: $\widetilde{u^{\prime \prime} v^{\prime \prime}}$ and $\widetilde{v^{\prime \prime} t^{\prime \prime}}$ profiles across the channel at different heights.

the location where the temperature boundary layer begins. As the boundary layer evolves, $\widetilde{v^{\prime \prime} v^{\prime \prime}}$ attains larger/smaller values close to the hot/insulated wall.

The Reynolds shear stress together with the stream-wise turbulent heat flux have significant impacts on $u^{\prime \prime} u^{\prime \prime}$ whose variations across the channel at different heights are shown in Figure 11(b). This can be understood by considering the production of $\widetilde{u^{\prime \prime} u^{\prime \prime}}$ whose major terms are $-2 \widehat{u^{\prime \prime} v^{\prime \prime}} \partial \widetilde{U} / \partial y$ and $2 g \beta \widehat{u^{\prime \prime} t^{\prime \prime}}$. In the inner boundary layer, due to the negative velocity gradient and positive shear stress, the former term is positive. However, the latter term is negative due to the negative $\widetilde{u^{\prime \prime} t^{\prime \prime}}$ (see Figure 12(a)). Nevertheless, as the magnitude of the production due to the shear stress is larger, the total production remains positive. In the outer boundary layer, where

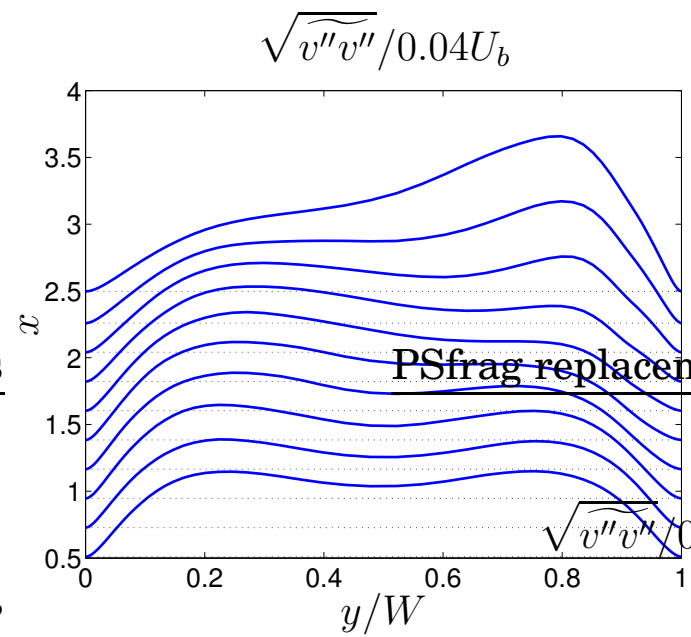

(a) Wall normal Reynolds stress.

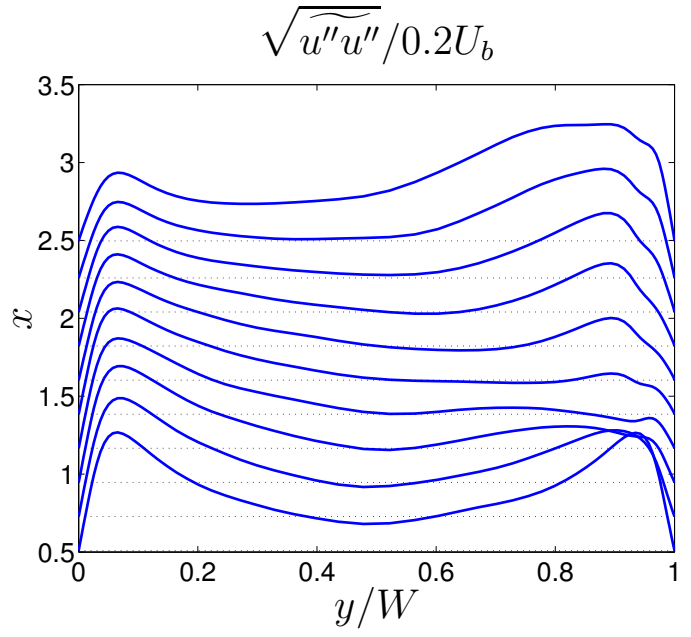

(b) Stream-wise Reynolds stress.

Figure 11: $\widetilde{v^{\prime \prime} v^{\prime \prime}}$ and $\widetilde{u^{\prime \prime} u^{\prime \prime}}$ profiles across the channel at different heights. 
$\widetilde{u^{\prime \prime} v^{\prime \prime}}$ and $\widetilde{v^{\prime \prime} t^{\prime \prime}}$ are negative and $\widetilde{t^{\prime \prime} t^{\prime \prime}}$, the velocity and temperature gradients are positive, $P_{u t}=-\widetilde{v^{\prime \prime} t^{\prime \prime}} \partial \widetilde{U} / \partial y-\widetilde{u^{\prime \prime} v^{\prime \prime}} \partial \widetilde{T} / \partial y+g \beta t^{\prime \prime} t^{\prime \prime}$ remains positive which results in a positive $\widetilde{u^{\prime \prime} t^{\prime \prime}}$. Consequently, in the outer layer, the positive and large $\widetilde{u^{\prime \prime} t^{\prime \prime}}$ makes the $\widetilde{u^{\prime \prime} u^{\prime \prime}}$ large in the boundary layer.

Figure 12(b) shows the trend of $\widetilde{t^{\prime \prime} t^{\prime \prime}}$ at different heights of the channel. It can be seen that as the boundary layer grows, $\widehat{t^{\prime \prime} t^{\prime \prime}}$ attains larger values owing to the negative $\widehat{v^{\prime \prime} t^{\prime \prime}}$ and positive temperature gradient which makes its production, $P_{t t}=$ $-2 v^{\prime \prime} t^{\prime \prime} \partial \widetilde{T} / \partial y$, to remain positive and increase along the channel as $\left|\widetilde{v^{\prime \prime} t^{\prime \prime}}\right|$ increases.

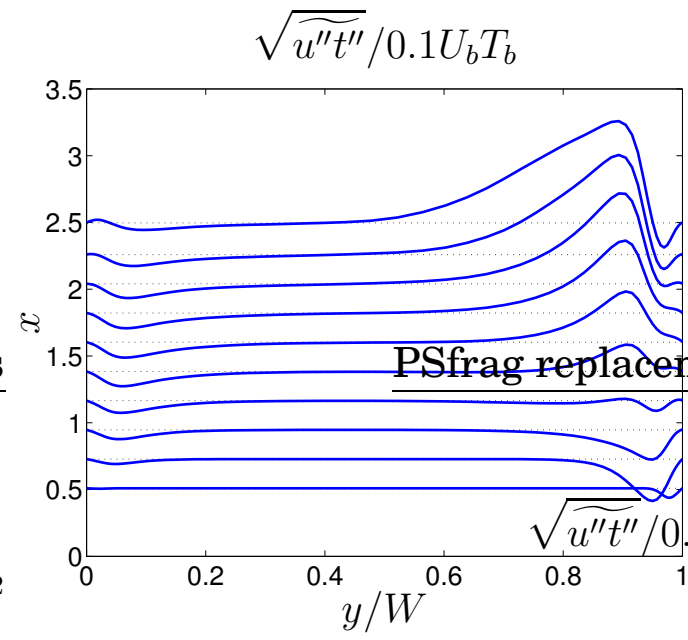

(a) Stream-wise turbulent heat flux.

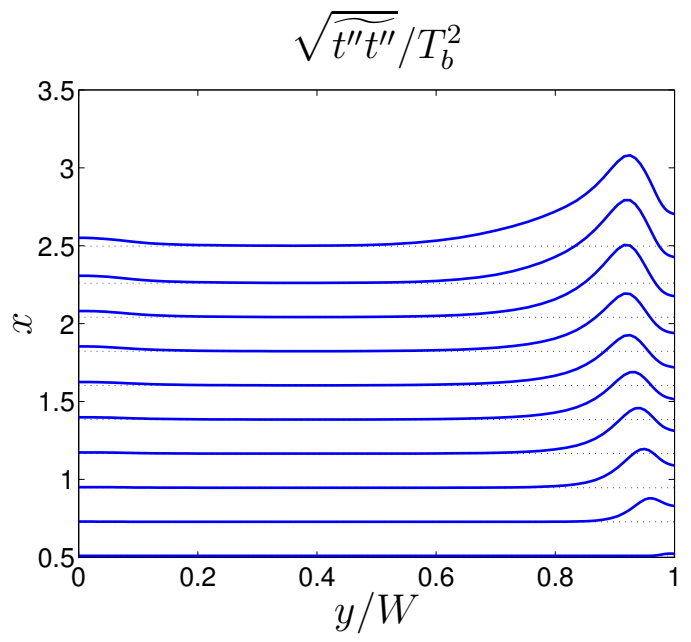

(b) Temperature fluctuations.

Figure 12: $\widetilde{u^{\prime \prime} t^{\prime \prime}}$ and $\widetilde{t^{\prime \prime} t^{\prime \prime}}$ profiles across the channel at different heights.

In turn, $\widetilde{t^{\prime \prime} t^{\prime \prime}}, \widetilde{v^{\prime \prime} t^{\prime \prime}}$ and $\widetilde{u^{\prime \prime} v^{\prime \prime}}$ affect the stream-wise turbulent heat flux. Positive $\widetilde{u^{\prime \prime} v^{\prime \prime}}$, results in a negative $\widetilde{u^{\prime \prime} t^{\prime \prime}}$ in a small region close to the wall as $P_{u t}$ is negative in that region. However, beyond this region, as the turbulent shear stress changes sign, $u^{\prime \prime} t^{\prime \prime}$ attains positive values in major portion of the boundary layer.

\section{Conclusions}

Boundary layer development in a vertical channel is studied using Large-Eddy simulation. Dynamic model is used as the SGS model. This model is able to capture the backscattered energy by yielding negative SGS-viscosity (see Figure 13).

Due to relatively high temperature differences, the full governing equations with variable properties are solved. The Boussinesq approximation yields different results, especially in case of the turbulence parameters. Mean flow parameters also show a thicker boundary layer in case of the full equations. Unfortunately, even full equations result in considerably different mean flow and turbulence parameters compared to the measurements. Considerably lower Nusselt number near the entrance region also suggests that the predicted low turbulence level at the inlet which is caused by the wiggle detector has resulted in a retarded boundary layer 


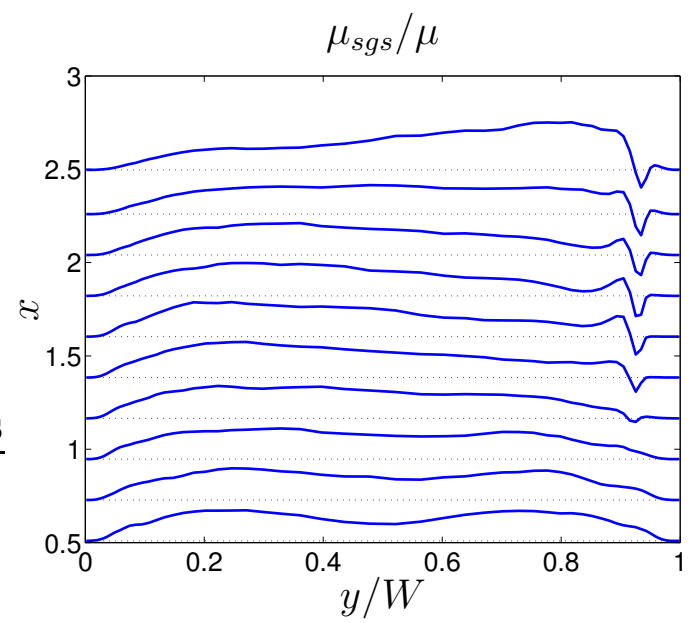

Figure 13: $\left\langle\mu_{\text {sgs }}\right\rangle$ profiles across the channel at different heights.

development and hence considerably different results. This anomaly may arise from the high frequency of the imposed signal at the inlet. Employing a coherent signal at the inlet may solve this anomaly. A fine mesh can also disengage the wiggle detector and lead to a less dissipative scheme.

\section{Acknowledgments}

This work is partly financed by the Swedish Research Council which is greatly acknowledged.

\section{References}

[1] K. Hanjalić. Achievements and limitations in modelling and computation of buoyant turbulent flows and heat transfer. In 10th Int. Heat Transfer Conference, Brighton, UK, 1994.

[2] T. Tsuji and Y. Nagano. Turbulence measurements in a natural convection boundary layer along a vertical flat plate. International Journal of Heat and Mass Transfer, 31(10):2101-2111, 1988.

[3] D. G. Barhaghi, L. Davidson, and R. Karlsson. Large eddy simulation of natural convection boundary layer on a vertical cylinder. International Journal of Heat and Fluid Flow, in print, 2006.

[4] L. Davidson, D. Čuturić, and S.-H. Peng. DNS in a plane vertical channel with and without buoyancy. In K. Hanjalić, Y. Nagano, and M.J. Tummers, editors, Turbulence Heat and Mass Transfer 4, pages 401-408, New York, Wallingford (UK), 2003. begell house, inc.

[5] J. Wang, J. Li, and J. D. Jackson. A study of the influence of buoyancy on turbulent flow in a vertical plane passage. International Journal of Heat and Fluid Flow, 25(3):420-430, 2004.

[6] M. Germano, U. Piomelli, P. Moin, and W. H. Cabot. A dynamic subgrid-scale eddy viscosity model. Physics of Fluids A, 3:1760-1765, 1991.

[7] S. Dahlström. Large Eddy Simulation of the Flow Around a High-Lift Airfoil. PhD thesis, Dept. of Thermo and Fluid Dynamics, Chalmers University of Technology, Göteborg, Sweden, 2003.

[8] L. Davidson and S.-H. Peng. Hybrid LES-RANS: A one-equation SGS model combined with a $k-\omega$ model for predicting recirculating flows. International Journal for Numerical Methods in Fluids, 43:1003-1018, 2003. 